\title{
HOM DAMPED 500 MHZ CAVITY DESIGN FOR 3RD GENERATION SR SOURCES*
}

\author{
F. Marhauser, E. Weihreter, BESSY II, Berlin, Germany \\ D.M. Dykes, P. McIntosh, CLRC, Daresbury, England
}

\begin{abstract}
Higher order mode (HOM) damped cavities are one option to avoid multibunch instabilities in modern synchrotron radiation (SR) sources, which can reduce their performance potential. Two different cavity shapes (spherical and cylindrical) both using ridged circular waveguides for HOM damping have been optimised to find a good compromise with respect to maximum fundamental mode shunt impedance and minimum HOM impedances. The properties of the two cavity designs are discussed and impedance thresholds for the excitation of multibunch instabilities are presented for several 3rd generation SR sources, which utilise $500 \mathrm{MHz}$ rf-systems. As a result of this numerical study the transverse and longitudinal HOM impedance can be reduced below threshold for most of these sources.
\end{abstract}

\section{INTRODUCTION}

Beam induced HOMs in rf cavities can severely deteriorate the photon beam quality of undulator dominated $3^{\text {rd }}$ generation SR sources by exciting multibunch instabilities, which potentially leads to emittance blow-up and/or an increase in energy spread with a consequent loss in brilliance. To reach the full performance capability it is essential to avoid such instabilities, e.g. by using HOM damped cavities. In the frame of an EC funded RTD project a normally conducting $500 \mathrm{MHz}$ cavity using circular waveguides for HOM suppression is under development especially for the use in SR sources. Circular waveguides offer considerable engineering and cost advantages as compared to rectangular waveguides. A fundamental mode frequency of $500 \mathrm{MHz}$ has been selected as this frequency became rather popular in the past for many low and medium energy sources.

Three tapered broadband circular waveguide to coaxial transitions (CWCT) with symmetrical ridges as described in [1] will be used to couple to the HOMs and to transfer their energy to matched external loads. For the present numerical study, however, homogenous double ridged waveguides with a perfect match at the end $(\mathrm{VSWR}=1)$ have been assumed to reduce the number of mesh points significantly. Only damping via the first propagating $\mathrm{TE}_{11}$-like waveguide mode is considered, as the future CWCTs show the same characteristic due to their geometry. The numerical method described in [2] was used, which is based on the 3D time domain solver (T3) of the MAFIA code [3] to evaluate the cavity impedance by a Fourier Transform of the computed wakefield. This method has been proved experimentally to give reliable results ([4], [2]).

\section{CAVITY CONFIGURATIONS}

To find out the best resonator geometry a cylindrically shaped $(C S)$ and a spherically shaped $(S S)$ re-entrant cavity with nose cones (beam pipe diameter $74 \mathrm{~mm}$ ) has been optimised. Figure 1 illustrates the layout of both cavities. The waveguides have a cutoff frequency of $650 \mathrm{MHz}$ and $670 \mathrm{MHz}$ for the $C S$ and $S S$ case respectively, sufficiently below the frequency of the first dangerous HOM, but sufficiently above the fundamental mode to avoid strong coupling. They are equally spaced in azimuth by $120^{\circ}$ to allow efficient coupling to HOMs with different polarisations. In the $S S$ cavity an additional port has been implemented for an iris rf-window which is assumed to contribute also to HOM damping, whereas in case of the $C S$ cavity a port for a coaxial rf window without any coupling to the HOMs has been modelled in. Additional ports are included for a tuner and a measuring probe.

To improve coupling to anti-symmetric $\mathrm{TM}_{0 \mathrm{mn}}$ like modes (odd $n$ ) the damping waveguides were inclined off the mid-plane towards one of the nose cones for the $S S$ cavity, and shifted parallel to the axis for the $C S$ cavity, one waveguide in opposite direction of the others.

A conceptual advantage of the CS cavity is the small insertion length as the damping waveguides are oriented perpendicularly to the cavity axis.
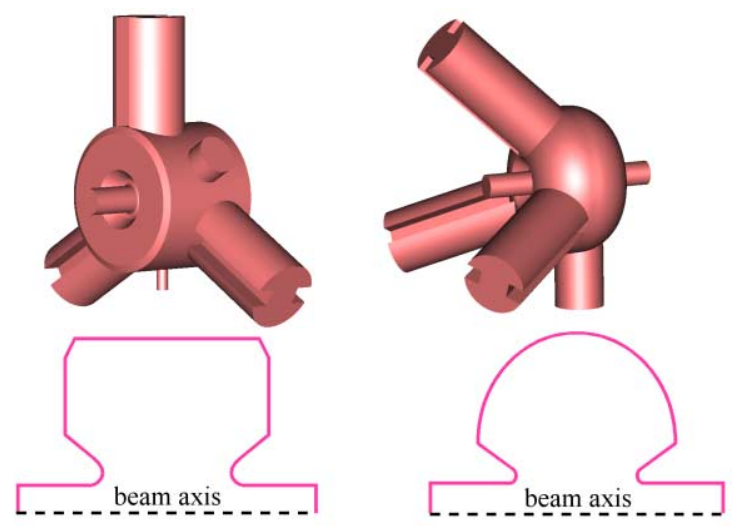

Figure 1: Top: Layout of the cylindrical $(C S)$ and the spherical ( $S S$ ) cavity. Bottom: cavity cross-sections without waveguides and ports.

\footnotetext{
* Work supported by the EC under contract no. HPRI-CT-1999-50011
} 


\section{RESULTS}

Both cavity configurations have been optimised by iteration of all relevant parameters: length and radius of the cavity, nose cone angle, accelerating gap length, waveguide cutoff-frequency, and position of the waveguide. The cavity impedances (i.e. impedance spectra normalised to the bunch spectrum) are shown in Figure 2 for the relevant frequency range below the beam pipe cutoff, indicating that the impedance can be reduced down to the level of $2 \mathrm{k} \Omega$ and $60 \mathrm{k} \Omega / \mathrm{m}$ for the longitudinal and transverse case respectively. The $C S$ cavity shows slightly larger peak values in longitudinal impedance and lower peak values in transverse impedance as compared to the $S S$ cavity. Evaluation of the fundamental mode shunt impedance was done in frequency domain (3D), with the result of an effective impedance of 4.5 and $4.7 \mathrm{M} \Omega$ for the $C S$ and $S S$ copper cavity respectively.
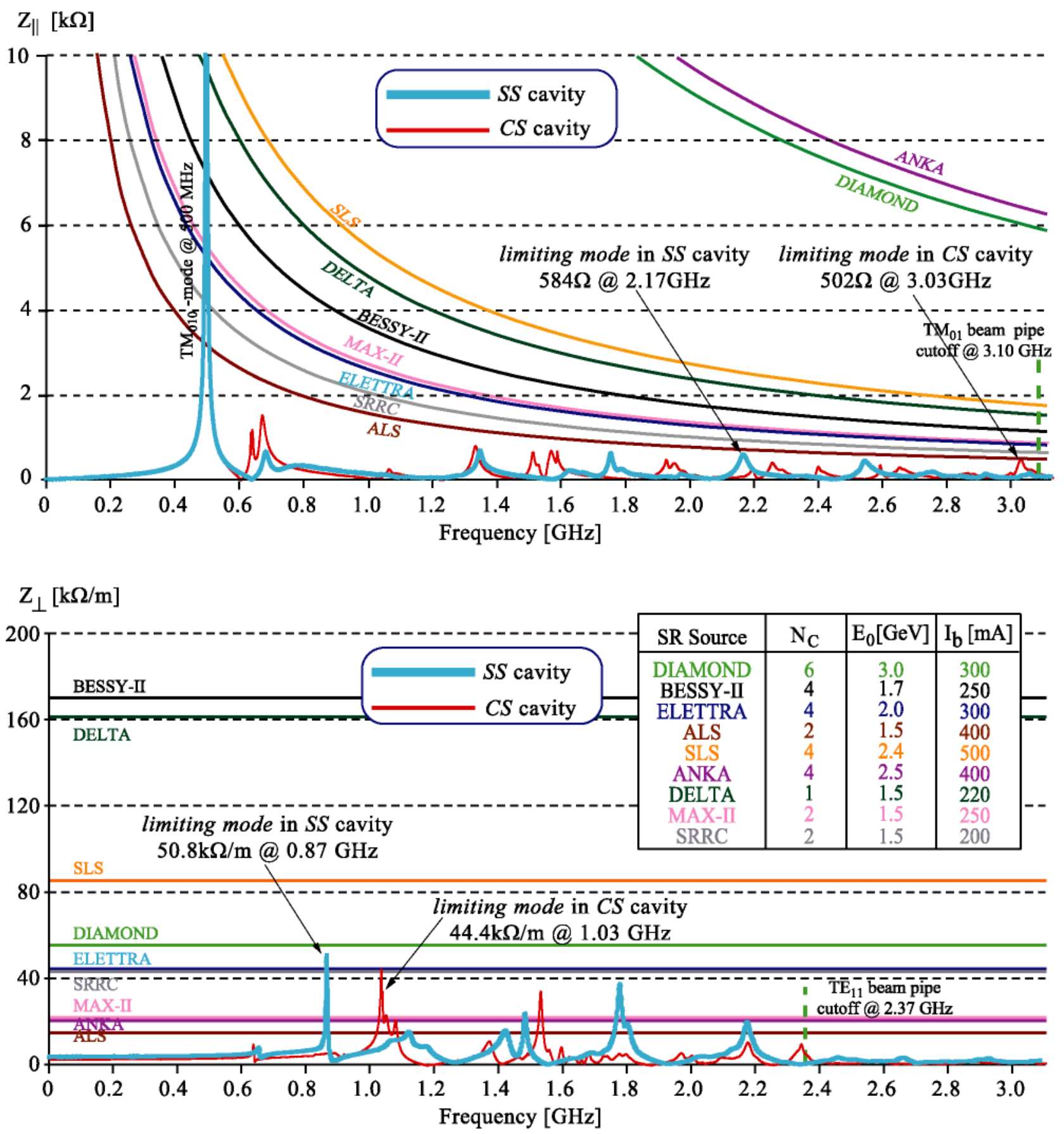

Figure 2: Longitudinal (top) and transverse impedance spectra (bottom) of the CS and SS cavity and impedance thresholds (see text) of various SR sources using $500 \mathrm{MHz}$ rf-systems. 
The field enhancement factor (defined by the ratio of the maximum electric field on the cavity walls to the effective field on the axis) has been evaluated in 2D by the SUPERFISH code [5] using about 250000 mesh points to obtain a reliable peak surface field independent of mesh size, with the result of 7.9 and 7.6 for the $C S$ and $S S$ cavity respectively. If validity of the Kilpatrick criterion [6] is assumed giving a breakdown limit of 21.3 MV/m at $500 \mathrm{MHz}$ for the electric surface field, a cavity voltage of $626 \mathrm{kV}$ and $611 \mathrm{kV}$ can be realised with the $C S$ and $S S$ cavity respectively. As this criterion is known to be too conservative, however, the layout of the final prototype cavity will be based on a cavity voltage of $850 \mathrm{kV}$ leading to a thermal power level around $80 \mathrm{~kW}$ for both cavity shapes.

The relevance of a given cavity impedance spectrum for the excitation of multibunch instabilities in a storage ring is best described by the threshold impedance, $\mathrm{Z}^{\text {thresh }}$, which can be obtained by equating the radiation damping time with the respective multibunch instability rise time, giving

$$
\begin{aligned}
& Z_{\|}^{\text {thresh. }}=\frac{1}{N_{C}} \cdot \frac{1}{f_{\|, H O M}} \cdot \frac{2 \cdot E_{0} \cdot Q_{s}}{I_{b} \alpha \tau_{s}} \\
& Z_{x, y}^{\text {thresh. }}=\frac{1}{N_{C}} \cdot \frac{2 \cdot E_{0}}{f_{r e v} I_{b} \beta_{x, y} \tau_{x, y}}
\end{aligned}
$$

for the longitudinal and the transverse case respectively. Here the conservative assumption has been made, that every HOM coincides with an instability driving beam frequency and that the $\mathrm{N}_{C}$ cavities have identical impedances ( $E_{0}$ beam energy, $I_{b}$ average beam current, $Q_{s}$ synchrotron tune, $\alpha$ momentum compaction, $\mathrm{N}_{\mathrm{C}}$ number of cavities, $f_{\|, H O M}$ long. HOM frequency, $f_{\text {rev }}$ revolution frequency, $\tau_{x, y, s}$ damping times, $\beta_{x, y}$ beta function at the cavity). With these expressions the threshold impedances have been calculated for several SR sources (with $500 \mathrm{MHz}$ rf systems) and plotted together with the HOM impedances for the optimised $C S$ and $S S$ model cavities in Figure 2. As a result for both cavities all considered machines can be operated without being affected by longitudinal multibunch oscillations, whereas a few rings may still be subject to transverse multibunch instabilities under these rather conservative assumptions. However, to control resistive wall instabilities, many modern SR sources use transverse feedback systems anyway, which may then stabilize also the remaining transverse oscillations.

According to eq. (1) the longitudinal impedance threshold is inversely proportional to the respective HOM frequency, $\mathrm{f}_{\|, \mathrm{HOM}}$. Thus, in contrast to the transverse case, the performance limiting mode in the longitudinal case does not necessarily correspond with the mode of highest impedance. Table 1 summarises these limiting modes for the $C S$ and the $S S$ cavity in comparison with the PEP II [4] and the DAФNE [7] cavity, normally conducting resonators, which have been developed several years ago for $\mathrm{e}^{+} \mathrm{e}^{-}$collider rings using rectangular waveguides for HOM-damping.

Table 1: TM010-mode frequency, shunt impedance and limiting modes (as calculated in T3) in different HOMdamped cavities

\begin{tabular}{|c|c|c|c|c|}
\hline cavity & $\begin{array}{c}\mathrm{f} \\
{[\mathrm{MHz}]}\end{array}$ & $\begin{array}{c}\mathrm{R}_{\mathrm{Sh}} \\
{[\mathrm{M} \Omega]^{* *}}\end{array}$ & \multicolumn{2}{|c|}{ performance limiting modes } \\
\cline { 4 - 6 } & longitudinal case & transv. case \\
\hline DAФNE & 368.3 & 2 & $2.1 \mathrm{k} \Omega @ 2.61 \mathrm{GHz}{ }^{* *}$ & $>80 \mathrm{k} \Omega / \mathrm{m}^{* * *}$ \\
\hline PEP-II & 476 & 3.8 & $2.6 \mathrm{k} \Omega @ 2.48 \mathrm{GHz}$ & $144 \mathrm{k} \Omega / \mathrm{m}$ \\
\hline$S S$ & 500 & 4.7 & $0.6 \mathrm{k} \Omega @ 2.17 \mathrm{GHz}$ & $51 \mathrm{k} \Omega / \mathrm{m}$ \\
\hline$C S$ & 500 & 4.5 & $0.5 \mathrm{k} \Omega @ 3.03 \mathrm{GHz}$ & $44 \mathrm{k} \Omega / \mathrm{m}$ \\
\hline \multicolumn{2}{|c|}{${ }^{* *} \mathrm{R}_{\mathrm{Sh}}=\mathrm{U}_{\text {eff }}{ }^{2} /\left(2 * \mathrm{P}_{\text {Loss }}\right)$ for copper, ${ }^{* * *}$ mode not fully resolved }
\end{tabular}

Both cavity configurations presented here show a significantly lower impedance for the limiting modes compared with the collider cavities, giving improved instability thresholds by about a factor of 3 and 2 in the longitudinal and in the transverse case respectively.

\section{CONCLUSION AND OUTLOOK}

It has been shown numerically that an impressive reduction in HOM impedance can be achieved for the two cavities studied using circular waveguides for HOM damping, which would allow to operate many existing SR sources below their thresholds for multibunch instabilities. The present cavity concepts also offer a good fundamental mode shunt impedance and a relatively simple mechanical layout with the potential of savings in operation and investment costs.

A thermal analysis is under way to study the high power performance of such a cavity with the aim to build a functional prototype cavity, which will be tested in the DELTA ring.

\section{ACKNOWLEDGEMENTS}

We would like to thank R.A. Rimmer and R. Boni for information concerning the PEP-II and DA $\Phi$ NE cavity.

\section{REFERENCES}

[1] E. Weihreter, S. Küchler, Y.C. Tsai, K.R. Chu, Proc. of EPAC 1998, vol. 2, p. 2065

[2] F. Marhauser, E. Weihreter, D.M. Dykes, P. McIntosh, S.A. Pande, K.R. Chu, Y.C. Tsai, C. Wang, R.A. Rimmer, Proceedings of the EPAC 2000, Vienna Austria, p. 1702

[3] MAFIA Manual PC V.4, CST Company (1999)

[4] R.A. Rimmer, J.M. Byrd, D. Li, Physical Review Special Topics-Accelerator and Beams, vol. 3, 102001 (2000)

[5] K. Halbach, R.F. Holsinger, SUPERFISH, Published in Part.Accel. 7: 213-222, 1976

[6] W.D. Kilpatrick, LBL Report , Berkeley, UCRL2321-REV, Apr. 1957

[7] R. Boni, A. Galli, F. Marcellini, Proceedings of the EPAC 1996, vol. 3, p. 1979 